\title{
Numerical solutions of Schön-Klasens model for luminescence efficiency
}

\author{
Erdem Uzun a and Mehmet Emin Korkmaz \\ Karamanoglu Mehmetbey University, Faculty of Kamil Özdağ Science, Department of Physics, 70100, Karaman, Turkey
}

\begin{abstract}
Phosphors exhibit luminescence following irradiation and the absorption of energy depends upon the relative probabilities of the radiative and non-radiative transitions. In general, the luminescence efficiency of a phosphor is related to the probability of a luminescence transitions and probability of a non-radiative transitions. Several experimental measurements of the luminescence efficiency have shown it to be strong temperature dependent over given temperature ranges. In literature, Schön-Klasens model has been offered to explain the temperature dependence of luminescence efficiency. In this study, theoretical basis and some numerical solutions of the model was discussed. The brief information about mathematical principles of the model was given and differential equations expressing the charge carrier transitions were derived. Numerical solutions of the equations were performed by using Mathematica 8.0 computer code. All the analysed parameters were chosen realistically. According to the simulations, glow curves intensities were decreased by the appropriate $\eta_{\mathrm{e}}$ and $\eta_{\mathrm{h}}$ ratio.
\end{abstract}

\section{Introduction}

A number of models are available in the literature for the explanation of the thermoluminescence (TL) event. The theoretical explanation of TL is based on the electron band theory of an insulating or semiconducting solid. It consists of a set of localized energy levels in the forbidden band, which arises due the presence of impurities and other point defects. The energy levels act as traps and recombination centres in the TL process [15]. All TL phenomena are governed by the process of the electron hole recombination. It should be noted that rather complex processes are taking place in the traffic of charge carrier between trapping states and luminescent recombination centres. Almost all of TL models have been based on the consideration of charge release from electron trap only. In this paper, we assumed that not only electrons but also holes are mobile in the same temperature interval. The model introduced originally by Schön and colleagues [6,7] and used by Klasens [8]. According to the model holes also contribute to TL emitting like electrons. Fig. 1 show that energy levels, charge carrier transitions and related parameters suggested by the Schön-Klasens model [4].

According to the model charge carrier traffic are given in Eq.1-4. The 4 equations set also describe the simultaneous release of holes during the thermal stimulation of the trapped electrons [1-4].

$$
\begin{aligned}
& d n / d t=-s_{\mathrm{e}} \mathrm{n} \exp \left(-E_{e} / k T\right)+\mathrm{A}_{\mathrm{te}} \mathrm{n}_{\mathrm{c}}(\mathrm{N}-\mathrm{n})-\mathrm{A}_{\mathrm{rh}} \mathrm{n}_{\mathrm{v}} \mathrm{n} \\
& d n_{c} / d t=s_{\mathrm{e}} \mathrm{n} \exp \left(-E_{e} / k T\right)-\mathrm{A}_{\mathrm{te}} \mathrm{n}_{\mathrm{c}}(\mathrm{N}-\mathrm{n})-\mathrm{A}_{\mathrm{re}} \mathrm{n}_{\mathrm{c}} \mathrm{m} \\
& d m / d t=-S_{\mathrm{h}} \mathrm{m} \exp \left(-E_{h} / k T\right)+\mathrm{A}_{\mathrm{th}} \mathrm{n}_{\mathrm{v}}(\mathrm{M}-\mathrm{m})-\mathrm{A}_{\mathrm{re}} \mathrm{n}_{\mathrm{c}} \mathrm{m}
\end{aligned}
$$

$$
d n_{v} / d t={ }_{S_{\mathrm{h}}} \mathrm{m} \exp \left(-E_{h} / k T\right)-\mathrm{A}_{\mathrm{th}} \mathrm{n}_{\mathrm{v}}(\mathrm{M}-\mathrm{m})-\mathrm{A}_{\mathrm{rh}} \mathrm{n}_{\mathrm{v}} \mathrm{n}
$$

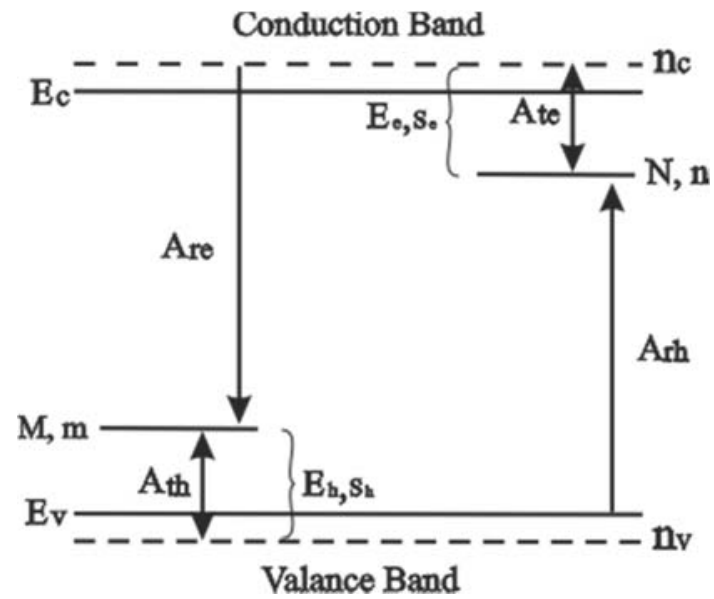

Figure 1. Generalized energy levels scheme and allowed transitions for Schön - Klasens model [4].

In here, the instantaneous concentration of electrons in the conduction band is denoted by $\mathbf{n}_{\mathbf{c}}\left(\mathrm{m}^{-3}\right)$ and that of holes in the valence band by $\mathbf{n}_{\mathbf{v}}\left(\mathrm{m}^{-3}\right)$ respectively. $\mathbf{N}$ $\left(\mathrm{m}^{-3}\right)$ denotes here the total concentration of electron trapping states which is a constant and $\mathbf{n}\left(\mathrm{m}^{-3}\right)$ the instantaneous concentration of filled electrons trap which is a variable. $\mathbf{E}_{\mathbf{e}}(\mathrm{eV})$ and $\mathbf{s}_{\mathbf{e}}\left(\mathrm{s}^{-1}\right)$ are the activation energy and frequency factor of the electron trap, respectively, $\mathbf{k}$ is the Boltzmann constant $\left(\mathrm{eV} \mathrm{K}^{-1}\right)$ and $\mathbf{A}_{\mathrm{te}}\left(\mathrm{m}^{3} \mathrm{~s}^{-1}\right)$ is the trapping (re-trapping during heating) probability of electrons from the conduction band. $\mathbf{M}\left(\mathrm{m}^{-3}\right)$ denotes here the total concentration of hole trapping states which is a constant and $\mathbf{m}\left(\mathrm{m}^{-3}\right)$ the instantaneous concentration of filled holes trap which is a variable. $\mathbf{E}_{\mathbf{h}}(\mathrm{eV})$ and $\mathbf{s h}$

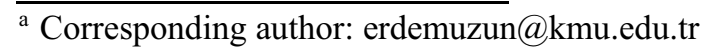


$\left(\mathrm{s}^{-1}\right)$ are the activation energy and frequency factor of the hole trap, respectively. $\mathbf{A}_{\mathrm{th}}\left(\mathrm{m}^{3} \mathrm{~s}^{-1}\right)$ is the probability of capturing hole in $M$, whereas $A_{\text {re }}\left(\mathrm{m}^{3} \mathrm{~s}^{-1}\right)$ is the recombination probability of free electrons with captured holes. A $\mathbf{A}_{\mathbf{r h}}\left(\mathrm{m}^{3} \mathrm{~s}^{-1}\right)$ is the recombination probability of free holes with captured electrons in electron trap.

At the same time, the equations to keep to the right neutralization condition expressed in Eq 5.

$$
d n / d t+d n_{c} / d t=d m / d t+d n_{v} / d t
$$

If all recombination events are radiative and produce photons and all photons are detected, TL glow curve is expressed by Eq.6. [1-4].

$$
I_{T L}=\eta_{\mathrm{e}} I_{T L n}+\eta_{\mathrm{h}} I_{T L h}
$$

For the equations set approximate solutions were given by Bräunlich and Scharmann [9]. These authors considered four extreme cases, involving the rates of electron and hole retrapping and their comparison with the corresponding recombination rates. The model also solved numerically by Mckeever et.al. [10] without any of the assumptions of the Bräunlich and Scharmann and reached the same conclusions. In addition to these the model was solved numerically by Uzun [11] for various trapping and recombination probabilities.

\section{Methodology}

In this study, we assumed that material irradiated before heating stage and has electrons in electron trap $\left(\mathrm{n}_{\mathrm{o}}\right)$ and holes in hole trap $\left(\mathrm{m}_{\mathrm{o}}\right)$. In this case it is important assumption that there are not any charge carriers in the conduction and valence bands. This is followed by a heating stage. During this stage $\mathrm{M}$ and $\mathrm{N}$ are assumed to be rather far from the valence band and conduction band, respectively. Electrons from $\mathrm{N}$ may be thermally released into the conduction band and then either re-trap in $\mathrm{N}$ or recombine with holes in $\mathrm{M}$. At the same time holes from $M$ may be thermally released into the valence band and then either re-trap in $\mathrm{M}$ or recombine with electrons in $\mathrm{N}$. For a given set of trapping parameters, differential equations governing the process during the excitation stage were numerically solved by using a special code in the Mathematica 8.0 [12] computer program with an explicit Runge-Kutta method [13]. During the solutions temperature was changing with a constant heating rate $(\beta)$ and therefore instantaneous temperature is expressed by Eq. 7.

$$
T=\mathrm{T}_{\mathrm{o}}+\beta \mathrm{t}
$$

Where $\mathbf{T}_{\mathbf{0}}$ is the initial temperature at the beginning of heating stage and $\mathbf{t}$ is the time (s). Both recombination into $\mathrm{N}$ and $\mathrm{M}$ are considered to be radiative, but separable. Thus, the intensity in photons per $\mathrm{m}^{3}$ per second of one spectral component of TL is proportional to the rate of change of $\mathrm{N}$, i.e. Eq. 1 and the second spectral component is assumed to be proportional to the rate of change of $\mathrm{M}$, namely, Eq. 3. The shape, position and intensity of the glow curve are related to a various trapping parameters of the trapping states responsible for the TL emission.

\section{Results}

Thermoluminescence intensities come from only electrons and only holes movement were determined and glow curve was calculated. Results are given in Fig. 2 and parameters used in the simulations were given in Table 1. It can be seen from the Fig. 2 that contributions of the electrons and holes on glow curve are the same for the given trap parameters in Table 1.

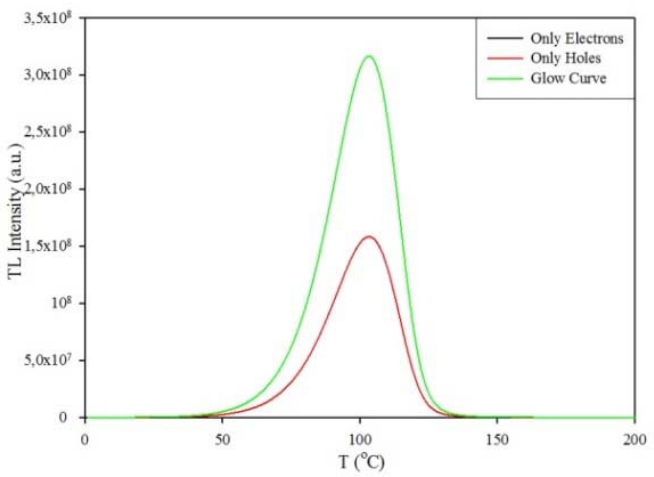

Figure 2. Thermoluminescence intensity comes from only electrons (black line), holes (red line) and glow curve (green line)

Table 1. Trap parameters for Fig.2

\begin{tabular}{|c|c|}
\hline Parameters & Values \\
\hline $\mathrm{E}_{\mathrm{e}}=\mathrm{E}_{\mathrm{h}}(\mathrm{eV})$ & 1.00 \\
\hline $\mathrm{Se}_{\mathrm{e}}=\mathrm{Sh}_{\mathrm{h}}\left(\mathrm{s}^{-1}\right)$ & $10^{12}$ \\
\hline $\mathrm{M}=\mathrm{N}\left(\mathrm{cm}^{-3}\right)$ & $10^{10}$ \\
\hline $\mathrm{m}=\mathrm{n}\left(\mathrm{cm}^{-3}\right)$ & $10^{10}$ \\
\hline $\mathrm{A}_{\mathrm{te}}=\mathrm{A}_{\mathrm{th}}$ & $10^{-9}$ \\
\hline $\mathrm{A}_{\mathrm{re}}=\mathrm{A}_{\mathrm{rh}}$ & $10^{-7}$ \\
\hline$\eta_{\mathrm{e}}=\eta_{\mathrm{h}}$ & 1.00 \\
\hline
\end{tabular}

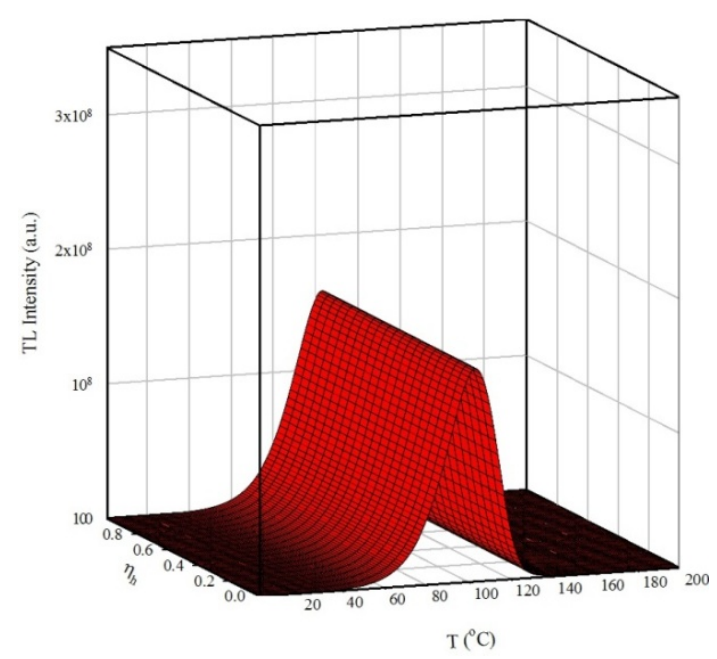

Figure 3. Contributions of the electrons on glow curve for different $\eta_{\mathrm{h}}$ values. 
The effect of the radiative recombination ratios of the electrons and holes on glow curves are show in Fig.3-5. Trap parameters used in the simulations were given in Table 2.

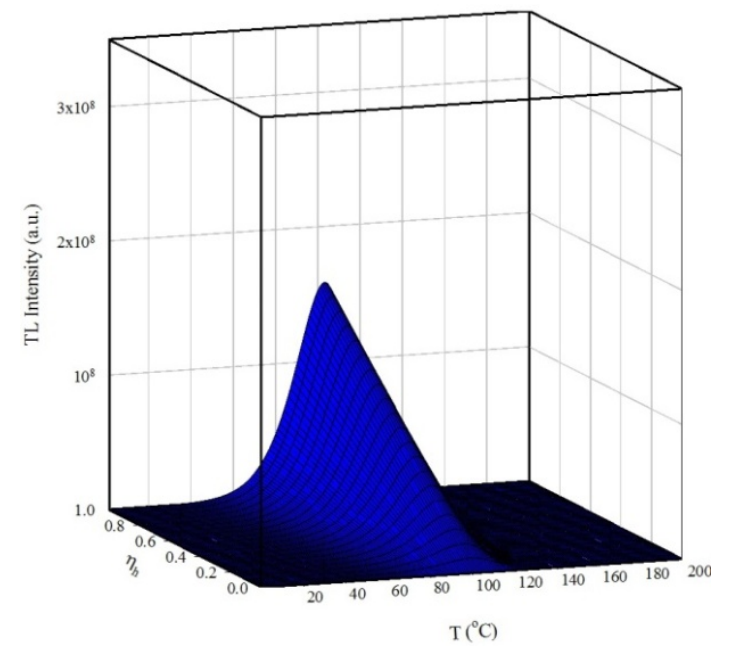

Figure 4. Contributions of the holes on glow curve for different $\eta_{\mathrm{h}}$ values.

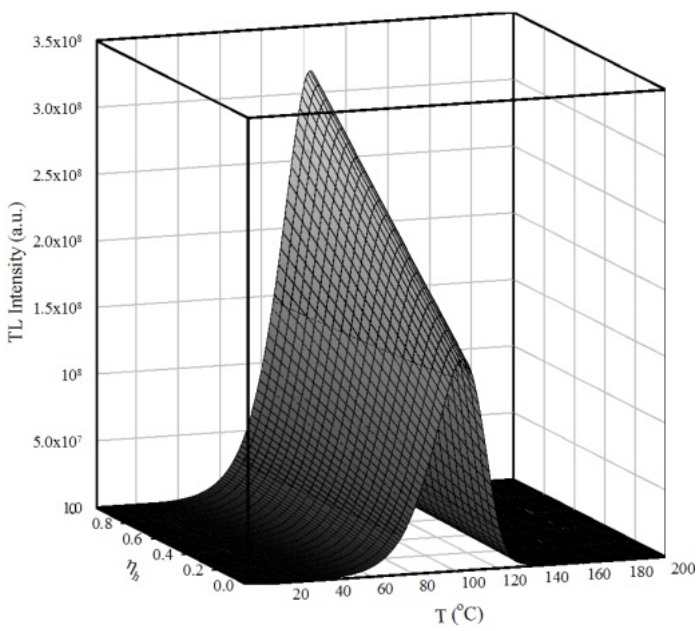

Figure 5. Glow curves for different $\eta_{\mathrm{h}}$ values.

Table 2. Trap parameters for Fig.3-5.

\begin{tabular}{|c|c|}
\hline Parameters & Values \\
\hline$\eta_{\mathrm{e}}$ & 1 \\
\hline$\eta_{\mathrm{h}}$ & $1.00-0$ \\
\hline
\end{tabular}

The effect of the radiative recombination ratios of the electrons and holes on maximum thermoluminescence intensities of the glow curves are show in Fig.6.

\section{Conclusions}

In this study Schön-Klasens model has been solved by numerically. In the solutions $\eta_{\mathrm{e}}$ and $\eta_{\mathrm{h}}$ parameters were chosen as variables and others were constant. By using these parameters, Eq. 1 to Eq. 6 are solved by numerically but no simplifying assumptions had been made.

Simulations show that according to Schön-Klasens model, in which electron and hole can be released by thermally, thermoluminescence glow curve is shaped by charge carrier movement resulting recombination. This process is different from the other models' process because, now hole is not a stable charge carrier.

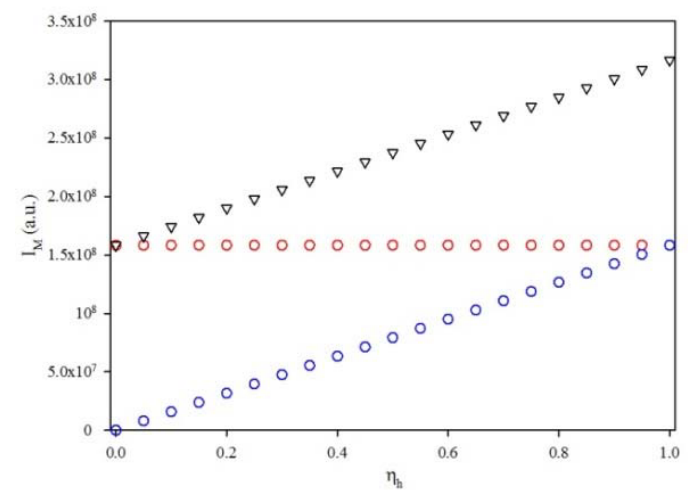

Figure 6. The effect of the $\eta_{\mathrm{h}}$ on $\mathrm{I}_{\mathrm{M}}$. Maximum intensities of the glow curve, electron and hole contributions are shown as black triangle, red and blue circles, respectively.

According to simulations;

i. When $E_{e}=E_{h}$ and $\eta_{\mathrm{e}}=\eta_{h}$, glow curve is shaped by both electrons and holes movement. In addition to this, contributions of them to glow curve are the same ratios.

ii. When $E_{e}=E_{h}$ and $\eta_{e}>\eta_{h}$, glow curve shaped by both electrons and holes movement. But in this case contribution of electrons is bigger than holes depending on $\eta_{\mathrm{e}} / \eta_{\mathrm{h}}$ ratio.

iii. When $\eta_{\mathrm{h}}=0$, glow curve is shaped by only electrons.

iv. When $\eta_{\mathrm{e}}=0$, glow curve is shaped by only holes.

v. When $\eta_{\mathrm{e}}=1$, it means that all electron-hole (electron is mobile and hole is still) recombination are radiative and vice versa if $\eta_{\mathrm{e}}=0$.

\section{References}

1. R. Chen, S.W.S. McKeever. Theory of Thermoluminescence and Related Phenomena (Word Scientific, Singapore, 1997)

2. R. Chen, D.J. Lockwood, J. Electrochem. Soc., 1499 (2002)

3. C. Furetta, Handbook of Thermoluminescence (Word Scientific, New Jersey, 2003)

4. S.W.S. Mckeever, R. Chen, Rad. Meas., 27 5/6 (1997)

5. S.W.S. McKeever, Thermoluminescence of Solids (Cambridge University Press, London, 1985)

6. N. Riehl, M. Schön, Z. Physik A, 114, 11-12 (1939)

7. M. Schön, Z. Physik A, 119, 7-8 (1942)

8. H.A. Klasens, Nature, 158 (1946)

9. P. Bräunlich, A. Scharmann, Phys. Status Solidi (b) 18 (1966)

10. S.W.S. Mckeever, et al., Phys. Rev. B, 326 (1985)

11. E. Uzun, JCBPS C, 52 (2015)

12. Wolfram Mathematica 8, Wolfram Research Inc., Version Number 8.0.0.0, Platform Microsoft Windows(64), Registered Org: Karamanoğlu Mehmetbey University.

13. https://reference.wolfram.com/language/tutorial/NDS olveExplicitRungeKutta.html 